\title{
BMJ Open Osseointegrated reconstruction and rehabilitation of transtibial amputees: the Osseointegration Group of Australia surgical technique and protocol for a prospective cohort study
}

\author{
Russel Haque, ${ }^{1,2}$ Shakib Al-Jawazneh, ${ }^{1,3}$ Jason Hoellwarth, ${ }^{1}$ \\ Muhammad Adeel Akhtar, ${ }^{4}$ Karan Doshi, ${ }^{1}$ Yao Chang Tan, ${ }^{5}$ William Yenn-Ru Lu (D) , \\ Claudia Roberts, ${ }^{2}$ Munjed Al Muderis ${ }^{1,2}$
}

To cite: Haque $\mathrm{R}$, AlJawazneh S, Hoellwarth J, et al. Osseointegrated reconstruction and rehabilitation of transtibial amputees: the Osseointegration Group of Australia surgical technique and protocol for a prospective cohort study. BMJ Open 2020;10:e038346. doi:10.1136/ bmjopen-2020-038346

- Prepublication history for this paper is available online To view these files, please visit the journal online (http://dx.doi. org/10.1136/bmjopen-2020038346).

Received 07 March 2020 Revised 25 August 2020 Accepted 26 September 2020

D) Check for updates

(C) Author(s) (or their employer(s)) 2020. Re-use permitted under CC BY-NC. No commercial re-use. See rights and permissions. Published by BMJ.

For numbered affiliations see end of article.

\section{Correspondence to} Dr William Yenn-Ru Lu; research@osseointegrationaust ralia.com.au

\section{ABSTRACT}

Introduction Lower extremity amputation uniformly impairs a person's vocational, social and recreational capacity. Rehabilitation in traditional socket prostheses (TSP) is associated with a spectrum of complications involving the socket-residuum interface which lead to reduced prosthetic use and quality of life. Osseointegration has recently emerged as a novel concept to overcome these complications by eliminating this interface and anchoring the prosthesis directly to bone. Though the complications of TSPs affect both transfemoral and transtibial amputees, Osseointegration has been predominantly performed in transfemoral ones assuming a greater benefit/risk ratio. However, as the safety of the procedure has been established, we intend to extend the concept to transtibial amputees and document the outcomes.

Methods and analysis This is protocol for a prospective cohort study, with patient enrolment started in 2014 and expected to be completed by 2022. The inclusion criteria are age over 18 years, unilateral, bilateral and mixed transtibial amputation and experiencing socket-related problems. All patients receive osseointegrated implants, the type of which depend on the length of the residuum and quality of bone, which are press-fitted into the residual bone. Objective functional outcomes comprising 6-Minute Walk Test, Timed Up-and-Go test and K-level, subjective patient-reported-quality-of-life outcomes (Short Form Health Survey 36 , daily prosthetic wear hours, prosthetic wear satisfaction) and adverse events are recorded preoperatively and at postoperative follow-up intervals of 3, 6, 12 months and yearly, and compared with the preoperative values using appropriate statistical tests. Multivariable multilevel logistic regression will be performed with a focus to identify factors associated with outcomes and adverse events, specifically infection, periprosthetic fracture, implant fracture and aseptic loosening.

Ethics and dissemination The Ethics approval for the study has been received from the University of Notre Dame, Sydney, Australia (014153S). The outcomes of this study will be disseminated by publications in peer-
Strengths and limitations of this study

- This study will be the first major one to focus on transtibial osseointegration only and will have the largest cohort reported in literature so far.

- The findings of the study would assess whether osseointegration in transtibial amputees (TTA) is feasible in terms of risks and benefits and also make an important contribution to the otherwise limited literature regarding outcomes of osseointegration in lower extremity amputations.

- The data may also help provide a foundation for estimating societal impact of transtibial osseointegration, particularly the true economic impact as compared with traditional socket prostheses (TSP) by indirect means.

- It does not have a control group and therefore comparison of outcomes of transtibial osseointegration directly with TSP used by TTA is not possible.

- The study has a follow-up period of minimum 2 years, which does not allow the examination of longer term outcomes and risk of adverse events as well as long term survivorship.

reviewed academic journals and scientific presentations at relevant orthopaedic conferences.

\section{INTRODUCTION}

Amputation of a lower extremity not only causes changes in the anatomy and function of the limb but also almost inevitably results in major impairments of the person's vocational, social and recreational abilities and overall quality of life. ${ }^{1}$ The focus of management of extremity amputations has evolved over time due to advancement of medical technology from prevention of mortality to overcoming these impairments and improving quality of life. ${ }^{2}$ For centuries, the conventional way of 
rehabilitating such individuals has been via traditional socket-mounted prostheses (TSP), ${ }^{3}$ and despite significant technological innovations to both socket materials and design, there has been very little change to the overall prosthetic-residuum interface from a moulded compression cone to modern suction-based socket suspension. ${ }^{4}$

The use of TSP is associated with a spectrum of complications arising mainly out of the socket-residuuminterface that causes reduction in prosthesis use, ability to mobilise and quality of life. ${ }^{15-7}$ These include skin problems such as infections, and skin breakdown due to chronic irritation and thermal injury, ${ }^{8-11}$ mechanical problems such as suboptimal fit, pain and pistoning ${ }^{12}$ and problems with proprioception that leads to loss of balance and falling. ${ }^{13}$ Gait with a TSP has been found to be asymmetrical correlating with a weakness in the hip abductor muscles, which can explain the back pain and pain in other regions experienced by such users including ipsilateral and contralateral limb, buttocks, neck and shoulder. ${ }^{14}$ Socket prostheses users account for their poor quality of life mostly to physical disability, pain and decreased energy levels. ${ }^{515}$

In order to overcome these complications, a significantly different concept has emerged over the past two decades, which circumvents the socket-residuum-interface completely by anchoring the prosthesis directly to the bone, popularly known as osseointegration. ${ }^{16}$ It involves insertion of porous metal implant in the medullary cavity of the bone in a screw or press-fit technique, over which compact cortical bone grows without any intervening soft tissue in a short course of time, integrating the implant structurally and functionally to the bone. ${ }^{17}$

This integration of non-vital component into living bone was first discovered serendipitously in 1950s in rabbit models ${ }^{4}$ and has been well established in the field of dentistry for the treatment of edentulous jaws for many years with a 10-year survival of dental implants in mandibular bone of 95\% ${ }^{18-21}$ Since its first introduction in 1990s in individuals with amputations, osseointegration has been predominantly used for the treatment of individuals with transfemoral amputation demonstrating multiple potential advantages such as improved walking ability, daily prosthetic use, reduced energy consumption, sitting comfort and osseoperception. ${ }^{722} 23$ This results in improved mobility and quality of life for individuals with amputations. ${ }^{1} 72224$

Over the last few years multiple studies have been published investigating the safety of this procedure, especially in individuals with transfemoral amputations, as incorporating a metal implant into the bone, while having an open connection with the outside environment can give rise to substantial concerns regarding the risk of ascending infection and concomitant implant loosening or sepsis. ${ }^{25-31}$ Multiple studies reported that despite frequent colonisation around the skin-implant interface, the implant system caused few infections leading to disability or implant removal (average 4\%). ${ }^{25-31}$ Most encountered complications were soft tissue infections or redundancy of soft tissue possibly influenced by learning curve and iteration of surgical technique and implant design. ${ }^{25} 29$

Osseointegration has been predominantly used in transfemoral amputees (TFA) as compared with transtibial amputees (TTA), due to apparently greater benefit-risk ratio with the TFA being perceived to have more socket related problems and poorer mobility as compared with TTAs and the extent of risks or complications of the new procedure largely unknown. ${ }^{152-34}$ Due to the same reasons, commercial availability of approved standard implants for TFA only promoted its use. Furthermore, it is much easier to press-fit or insert a screw fixation implant in to a cylindrical cortical bone such as a femur as opposed to the reverse pyramid shaped cancellous bone of the proximal tibia. ${ }^{26}$ It is very challenging to press fit an implant into cancellous bone and achieve immediate stability. The same principles apply to a screw fixation device.

With the establishment of safety of this procedure in literature, there is enough justification now for its use in individuals with TTA. First, the prevalence of transtibial amputations is much higher than transfemoral amputations. ${ }^{35} 36$ Of these individuals using socket prostheses, $40 \%$ experience at least one skin problem, with the percentage substantially higher in individuals with TTA (TTA: $45.8 \%$, TFA: 20\%; OR: 4.1). Second, there is increased percentage of stump pain reported in patients with TTA. ${ }^{87}$ Third, suboptimal socket fit occurs in both individuals with TTA and with TFA (TTA: 59\%, TFA: $78 \%)^{38}$ and dissatisfaction with socket prostheses does not differ when comparing for level of amputation, with only $43 \%$ being satisfied with the comfort of their prosthesis. ${ }^{39-41}$ These problems are inherently linked to intolerance of the prosthesis ${ }^{12}$ and impact the ability of TTA to become independently mobile. ${ }^{42}$

Until recently, there is very little data assessing the protocol, techniques and results of osseointegration in individuals with TTA. Only few papers with very small case series have been published with variable results. ${ }^{26} 284344$

\section{STUDY OBJECTIVES}

The overall objective of this study is to assess the safety and efficacy of transtibial osseointegration procedure with at least 2-year follow-up and to compare the benefits and risks from preoperative status and with the previously reported outcomes for transfemoral osseointegration. Specifically, this would involve:

1. Assessing the objective functional outcomes with the 6-Minute Walk Test (6MWT), ${ }^{45}$ Timed Up-and-Go (TUG) ${ }^{46}$ and K-levels, ${ }^{47}$ compared with preoperative data and with outcomes of TFA.

2. Assessing the subjective patient-reported quality-of-life outcomes with the Short Form Health Survey 36 (SF$36),{ }^{48}$ stump pain, daily prosthetic wear hours and prothetic wear satisfaction compared with preoperative data and with outcomes of TFA. 
Table 1 Inclusion and exclusion criteria with reason

Criteria
Inclusion criteria
Age at least 18 years
Current unilateral, bilateral or mixed transtibial amputees
with significant dissatisfaction regarding prosthesis fit or
pain, mobility, or skin breakdown
Patients with a full lower limb but with pain, deformity or
weakness distal to the mid-tibia who desired amputation for
pain management or improved mobility following removal of
the deformed or weak joint and muscles

Patients with amputations who wished to try
osseointegration instead of a traditional socket prosthesis

Patient with sufficient resources and willingness to pursue surgery, postoperative rehabilitation and prosthesis procurement

\section{Exclusion criteria}

Active infection any location

Active malignancy or ongoing/planned treatment for malignancy at any location

Skeletal immaturity

\section{Reason}

Legal self-consent

Objective, identifiable deficit in current patient lifestyle

Objective, identifiable quality of life impairment that can be objectively improved by amputation, and patients likely would experience better rehabilitation with osseointegration than standard socket prosthesis

Honouring patient choice after an ethical, shared and sound decision making process

Rehabilitation and prosthesis fitting are all required for appropriate, safe improvement following osseointegration surgery

Unacceptably high and modifiable infection risk

High risk for infection, impaired biology for osseointegration, impaired patient stamina for rehabilitation

Unknown risk given the current knowledge of osseointegration outcomes and biological impact

Minimise risk of performing surgery for a patient whose expressed deficits are psychiatric-based instead of musculoskeletal-based, and thus unlikely to improve with surgery

Patients considered too medically ill, too muscularly weak or Avoid harming patients with surgery that may be either unlikely insufficiently dedicated to improve following osseointegration to benefit them or possibly pose a health risk

Insufficient remaining tibia length to accept an implant

Avoid performing surgery for a patient who would be unlikely to achieve successful bone ingrowth to the implant

Uncontrolled diabetes mellitus

Avoid unnecessary, modifiable risk for infection

Women currently or intending to become pregnant within the year following surgery

Unnecessary risk to foetus due to potential for falls or other unforeseen adverse events
3. Examining the prevalence of adverse events, including infection, revision surgery, fractures, aseptic loosening and implant failures, and comparing with the adverse events after TFA.

One of the primary objectives of this study is to identify the individual patient characteristics or factors that have a positive or negative influence in the outcomes mentioned earlier. This analysis in a regression model would help to identify the patients based on their characteristics who would be most or least benefitted with this novel procedure and who would be at a higher or lower risk of failure.

The other objective is to identify the rate of additional surgical interventions as well as to identify factors associated with further surgery, specifically for infection, periprosthetic fracture, implant fracture and aseptic loosening.

\section{METHODS AND ANALYSIS}

This is a prospective cohort study which is designed to assess the safety and efficacy of transtibial osseointegration procedure with a minimum of 2 years (range 2-8 years) follow-up.
Preliminary data and clinical experience has been obtained from an initial pilot study comprising 10 patients owing to absence of prior literature. Software $\mathrm{G}^{*}$ Power was used to calculate an a priori sample size. Considering SF-36 physical component score as primary outcome measure, the preoperative and 2-year postoperative scores were recorded. Comparing the means (37.62 and 44.83) and SDs (11.8 and 19.5) of these two groups respectively using Wilcoxon test, the effect size was calculated to be 0.36 and sample size was calculated to be 87 assuming $\alpha$ error to be 0.05 and in order to achieve a Power of $95 \%$. Considering a drop-out rate of $20 \%$, a final sample size of 109 was decided on. None of the patients of the pilot study have been included in this study due to absence of standard protocol.

The first patient enrolled in the study was in April 2014. Enrolment is ongoing at the time of publication of this paper, with 68 patients already enrolled and is expected to be completed by April 2022. The number of patients treated each year has shown a steep rising 


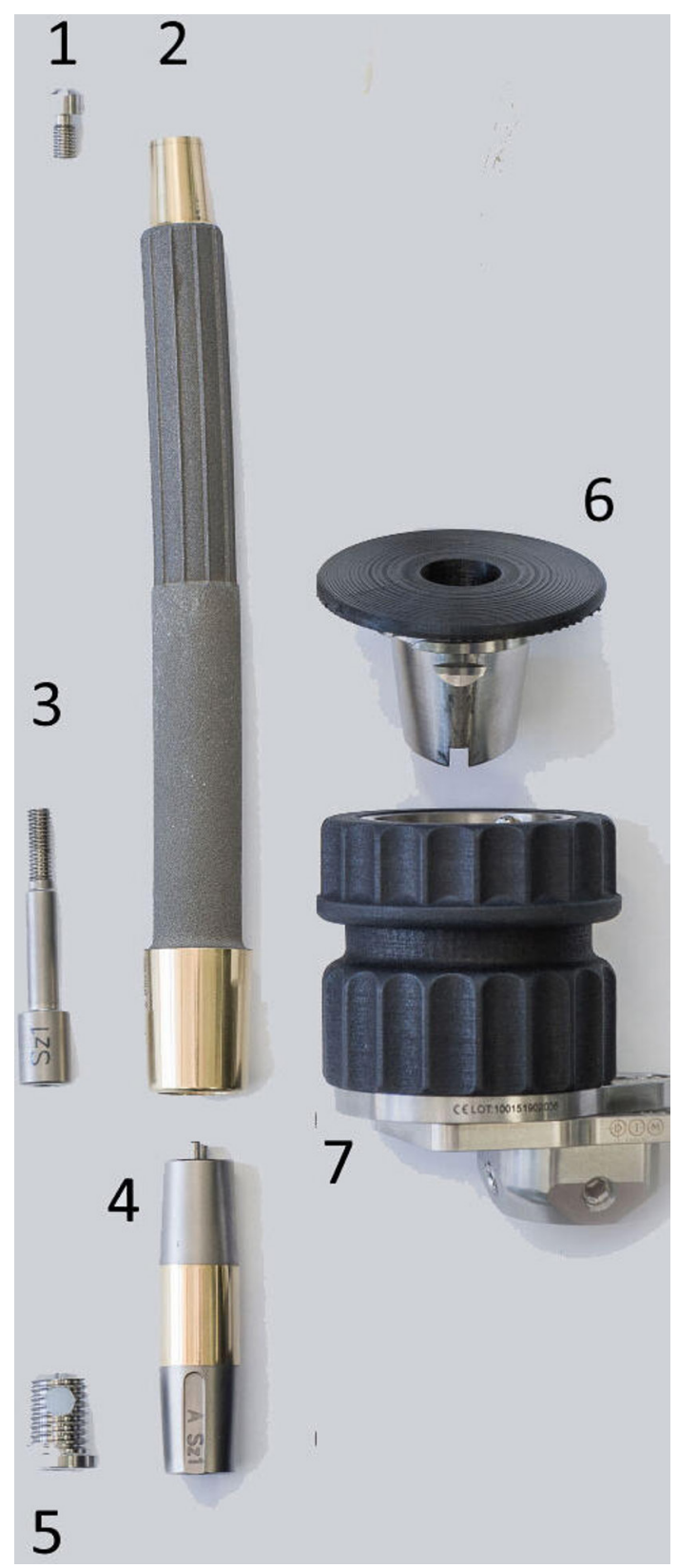

Figure 1 The standard implant for longer residuums. The parts include: 1, proximal cap screw; 2 , intramedullary body; 3 , internal safety screw; 4 , dual cone transcutaneous abutment adapter; 5 , permanent locking propeller screw; 6 , proximal connector; 7 , prosthetic connector.

trend with about 26 patients enrolled in the study last year.

\section{Patient selection}

Eligibility criteria

All participants gave their informed consent. Inclusion and Exclusion criteria along-with rationale are listed in table 1 .

\section{Patient recruitment}

Setting and patient screening

Our surgical practice is located in a private university hospital in a major urban city with full, modern medical

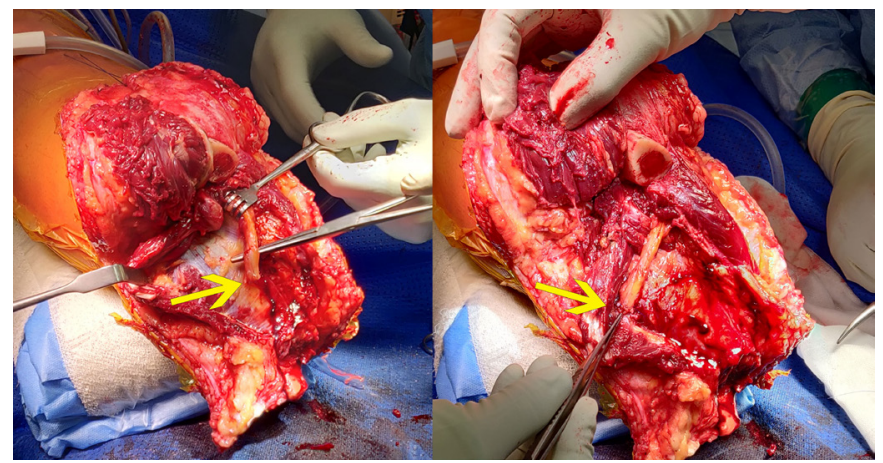

Figure 2 Targeted re-innervation of nerves (posterior tibial nerve highlighted) to surrounding muscular branches.

capabilities. Local patient referral can occur via the usual routes for our practice: from the general practitioner or by self-referral. Non-local patients within the country and international patients can also contact our office, as is typical already, and are encouraged to provide information for pre-evaluation. All patients being referred for, or requesting, osseointegration are required to complete an online patient screening form. Those patients fitting our inclusion and exclusion criteria are invited for in-person consultation. Patients who sustain acute traumatic injuries for which amputation is recommended can request osseointegration as primary management, either acutely or following the resolution of their acute injury.

\section{Patient enrolment}

All patients who complete the online patient screening form and fit the inclusion/exclusion criteria are evaluated in the multidisciplinary Limb Reconstruction Clinic. The typical medical team includes at least three orthopaedic surgeons with extensive limb reconstruction experience. Also, in attendance are a prosthetist and physiotherapist, to ensure the patient's disorders are not suitably improved by prosthesis adjustment or therapy. Patients are also evaluated by our psychiatrist to ensure absence of psychiatric conditions that can affect postoperative rehabilitation. For patients who have neuropathic pain or a history of narcotic or other pain-related medication use or abuse, a pain medicine consultation is

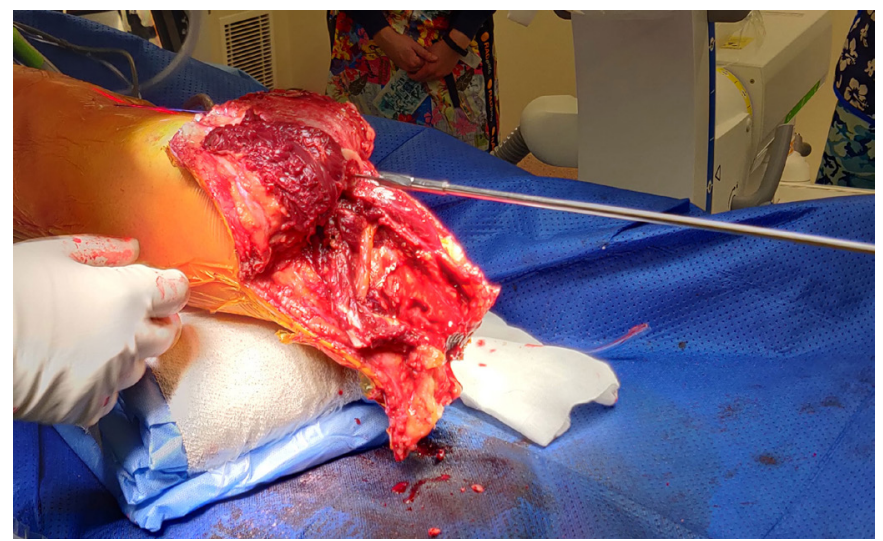

Figure 3 Reaming was done for longer residuums to $0.5 \mathrm{~mm}$ more than the diameter of implant expected to be used. 


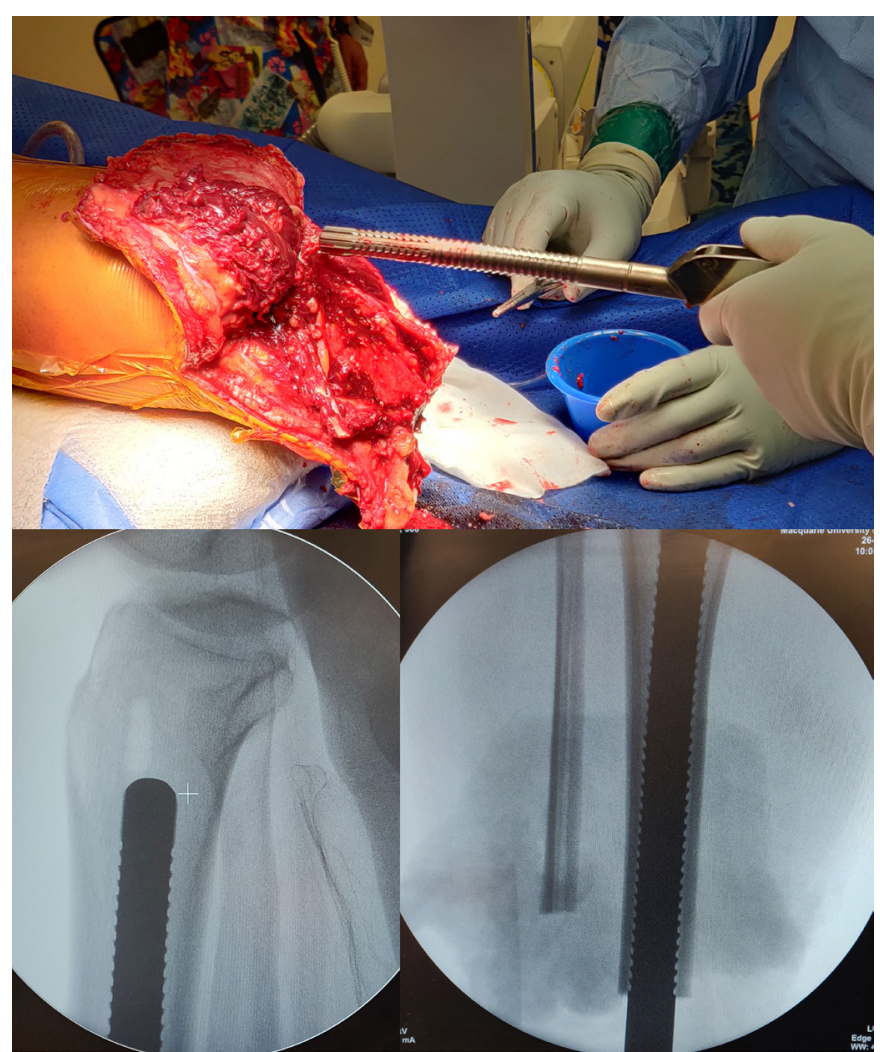

Figure 4 Broaching done under Image Intensifier guidance upto the desired size of implant for longer residuums.

required. All patients are counselled extensively by the team which includes a dynamic assessment and discussion of the benefits (mobility, quality of life, etc) as well as the risks (infection, fracture, further surgery including full removal or further amputation, etc) of osseointegration. The patients are fully explained about the relative novelty of this surgery and that the immediate and long-term risk/benefit profile is still not very well defined so that an ethical, sound and shared decision-making process is achieved. All patients who elect for osseointegration are informed their care is provided at the best clinical

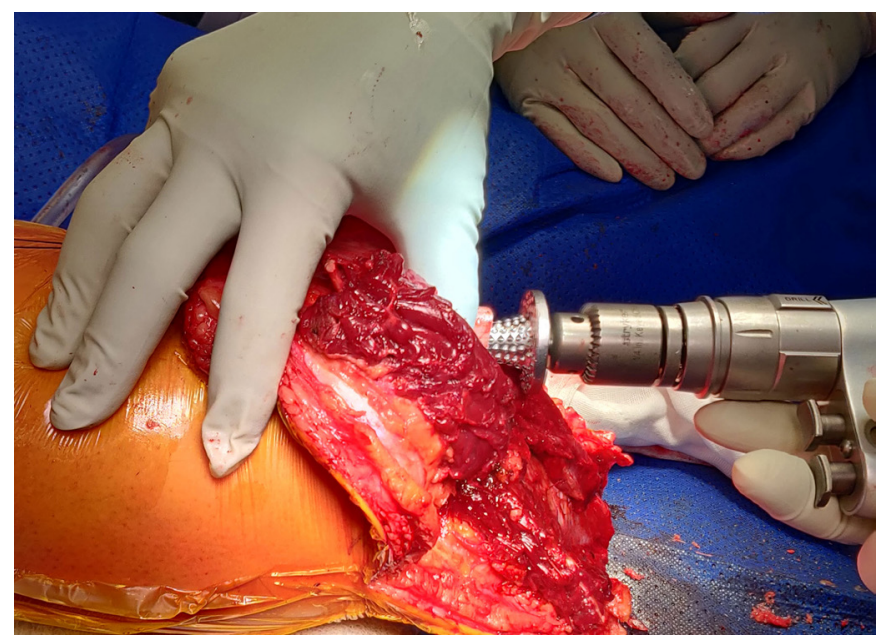

Figure 5 Face reaming done to smoothen the distal margins of the tibial stump.

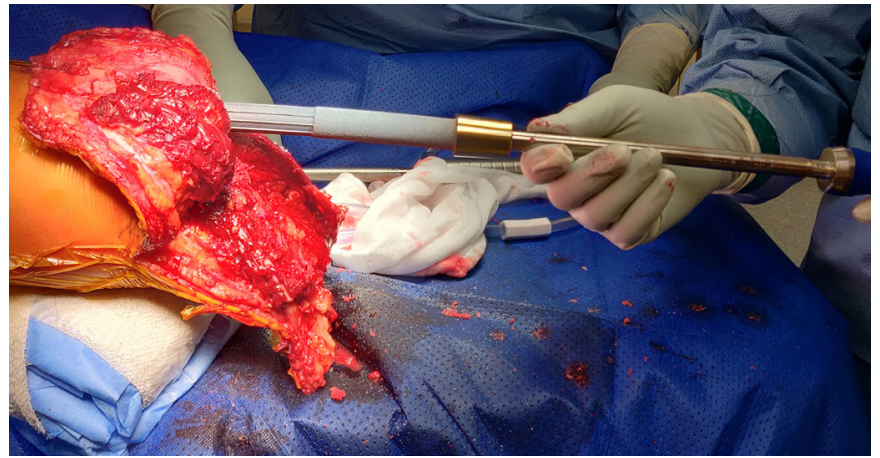

Figure 6 Final implantation of the definite intramedullary component.

judgement, but that they will be enrolled as part of a prospective and longitudinal study as described. There is no arbitrary treatment based on assignment into a treatment category. Implant selection and exact surgical technique is expressly tailored to each patient.

The time between patient enrolment and surgery will vary. Patients who have a traumatic injury and have inpatient consultation may have osseointegration the next day. Healthy patients with streamlined financial coverage and who are able to attain psychiatric evaluation quickly could have surgery within a week of consultation. For patients who do not have appropriate insurance coverage, there is a waiting period for the most appropriate coverage level of 1 year; and during that waiting time would be recommended to participate in prehabilitation exercises and have other perioperative optimisation performed.

\section{Potential selection bias}

One of the limitations of this study is possibility of selection bias to exclude low income patients. Osseointegration is an expensive surgery and thus is not covered by the standard government insurance for our country. It is covered by more premium insurance plans. Thus, we counsel patients to enrol in these top-level insurance plans so that not only will the surgery itself be provided but any additional surgery for an adverse event will be covered, so long as they maintain their coverage. Due to this limitation, the results of the study may not be generalisable to all countries and all populations.

\section{Study intervention}

\section{Preoperative management}

All the patients are assessed with Antero-posterior (AP) and lateral plain radiographs of the residuum to assess the bone quality and presence of any anomaly. Long leg standing radiographs are performed to assess the mechanical alignment of the lower limbs and to rule out pathologies in the contralateral limb. DEXA scans of the proximal femora and the spine to assess the bone mineral density which would help determine the speed of postoperative rehabilitation. Furthermore, CT scans of the residual bone are performed to plan for the type of implant and required size. 


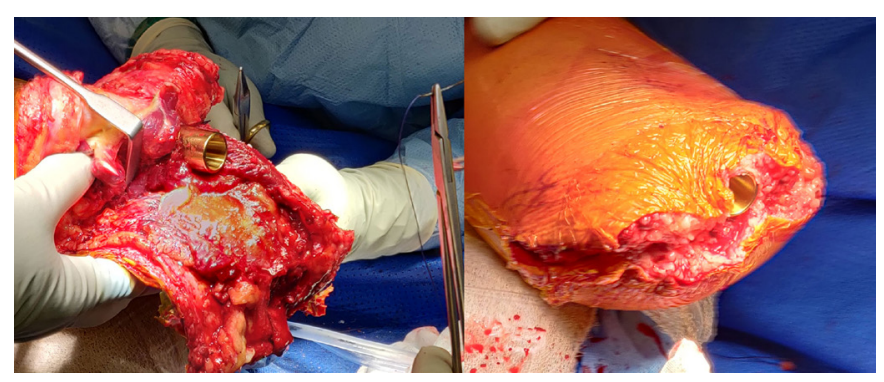

Figure 7 Closure of periosteum around the stump in a 'purse-string' fashion and the flaps around implant in 'fishmouth' manner.

\section{Osseointegration implant}

The transtibial osseointegration implant used by us for, was designed by senior author (MAM) into mainly two types. For longer residuums with sufficient cortical bone, a standard titanium implant which was machine manufactured of $160 \mathrm{~mm}$ length with plasma spraying on the surface was used (figure 1). Alternatively, for short residuums with metaphyseal bone a custom-made short stem titanium implant with coarser surface structure was either machine manufactured or three-dimensional printed. The surface of the implant is composed of a macroporous mesh-like structure allowing for bone ingrowth. Some implants contain longitudinal flanges for additional rotational stability. All implants are connected to a dual cone adapter with Morse-taper ends connecting the implant with the external prosthesis. The surface of the dual cone adapter is highly polished and coated with titanium-niobium oxide, an alloy known to have bacterial repellent properties, ${ }^{4}$ which also facilitates the excursion of the soft tissues and skin over it avoiding adhesions. A safety mechanism is built into the dual cone, with a safety pin that breaks to reduce the chance of periprosthetic fractures or implant breakage.



Figure 8 Attachment of extramedullary components.

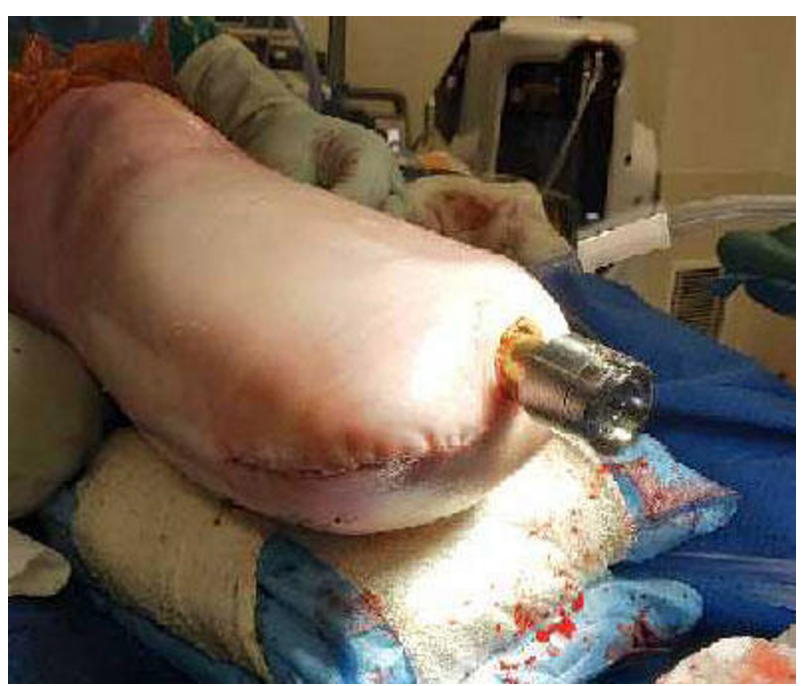

Figure 9 Final view of the closure of the stump.

\section{Surgical technique}

All patients receive an osseointegrated implant in a singlestage surgery. At the level of the distal stump, a horizontal elliptical incision is made, the amount of soft tissue and muscle tissue is minimalised and all nerves are sharply severed and vessels are ligated or cauterised until haemostasis is achieved. The saphenous, tibial and common peroneal nerves are re-innervated to surrounding muscle branches if symptoms of nerve pain or excessive phantom pain existed preoperatively (figure 2). Alternatively, the re-innervation of tibial and common peroneal nerves can be performed via a separate lateral distal thigh incision and posterior dissection.

Care is taken to preserve the periosteum at all times. If the distal end of the tibia needs to be re-cut, the periosteum is elevated and re-sutured to the end of the bone after using an oscillating saw for the distal tibia osteotomy. The fibula is usually cut $2-3 \mathrm{~cm}$ shorter than the tibia using the saw.

The intramedullary canal is prepared depending on the length of the residuum. If the amputation is at the diaphysial level with good cortical bone distally then reaming up to $0.5 \mathrm{~mm}$ larger than the definite implant anticipated to be used after cortical chatter is heard (figure 3 ) followed by sequential broaching up to the size of the desired implant (figure 4). If the tibial stump is at the metaphyseal level with poor quality bone then no reaming is done and only impaction broaching is performed usually stopping at $2 \mathrm{~mm}$ smaller than the definite size of the implant. Both reaming and broaching is performed under image intensifier guidance to ensure accurate positioning in the centre of the tibia on the AP and lateral planes. Finally, the distal edge of the tibia is smoothened with use of a face-reamer (figure 5).

Final implantation of the osseointegration intramedullary component is done using press-fit technique up to the subchondral bone of the proximal tibia (figure 6). To stabilise the implant in shorter residual stumps, multiple 
Transtibial Osseointegration Rehabilitation Protocol
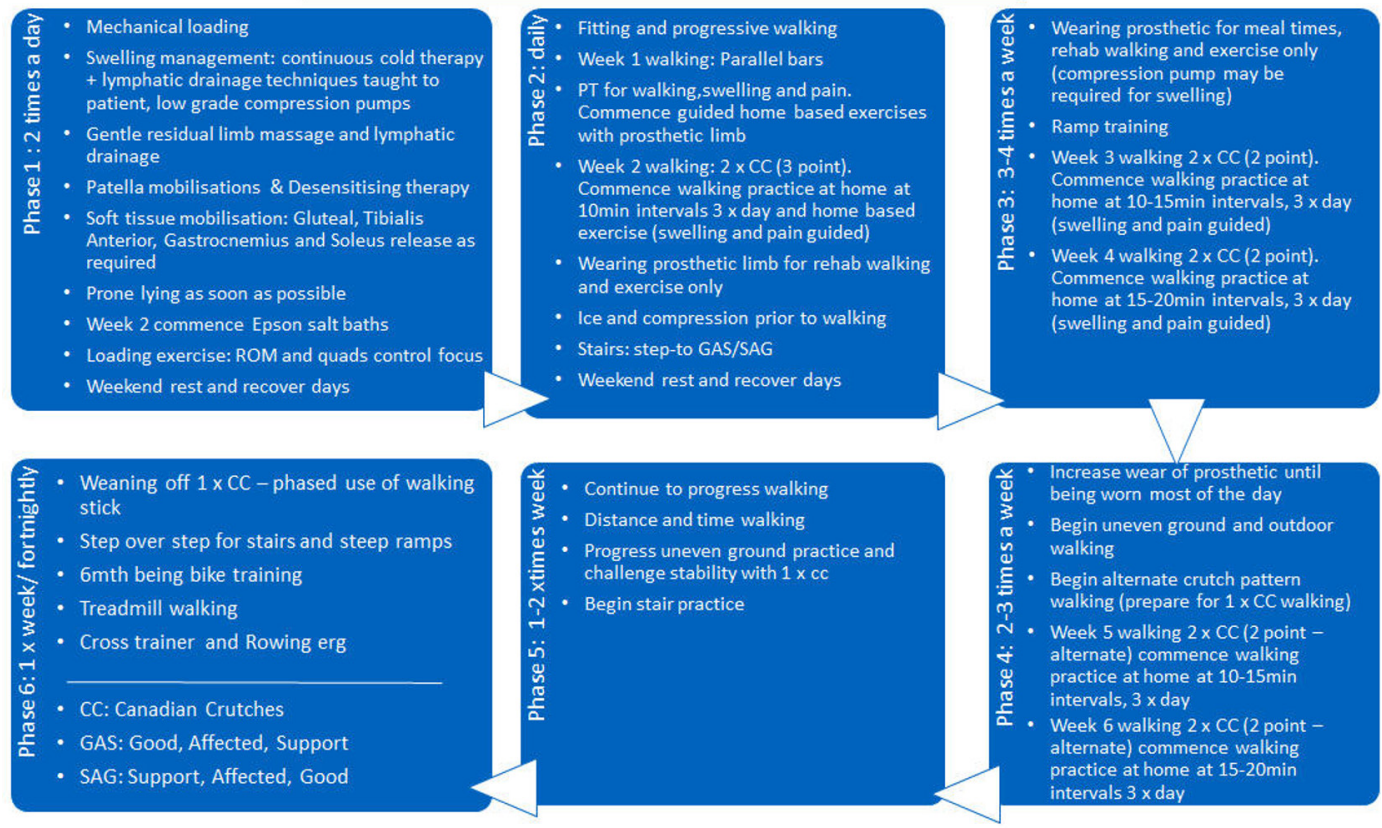

Fast loading (10 days -2 weeks loading (until $50 \%$ of body weight) $\rightarrow$ fitting $\rightarrow 6$ weeks $2 \times C C \rightarrow 6$ weeks $1 \times$ CC $\rightarrow$ progress off all aids) Phase $1:$ Week $1-2$, Phase 2: Week 3-4, Phase 3: Week 5-6, Phase 4: Week 7-8, Phase 5: 9-14, Phase 6: Week 14-24

Slow Loading ( 6 weeks loading, increase by $5 \mathrm{~kg}$ a week (until $50 \%$ of body weight) $\rightarrow$ fitting $\rightarrow 6$ weeks $2 \times \mathrm{CC} \rightarrow 6$ weeks $1 \times \mathrm{CC} \rightarrow$ progress off all aids) Phase 1: Week 1-6, Phase 2: Week 6-7, Phase 3: Week 8-9, Phase 4: Week 10-11, Phase 5: 12-17, Phase 6: Week 18-24

Figure 10 Transtibial osseointegration rehabilitation protocol.

locking screws were initially used, before it was abandoned due to increased risk of loosening and no added benefits.

Closure is initiated by suturing the fascia to the periosteum all around at the distal end of the tibial stump in a 'purse-string' fashion. This has not been described previously for tibias and is unique to our group. The anterior and posterior soft tissue sleeves are refashioned to remove subcutaneous fat. A flap is created-preferably anterior, to cover the end of the stump and to begin closure in layers. A sharp corer is used to make a stoma in the flap to communicate to the exact diameter of the implant, before progressing to close the rest of the wound in layers. Alternatively, the anterior and posterior flaps are closed around the implant in a 'fish-mouth' fashion (figure 7). After this step, the dual cone component of the osseointegration device is inserted and secured with an internal locking screw, followed by fixing the taper

Transtibial Osseointegration Physiotherapy Protocol

Knee Exercises
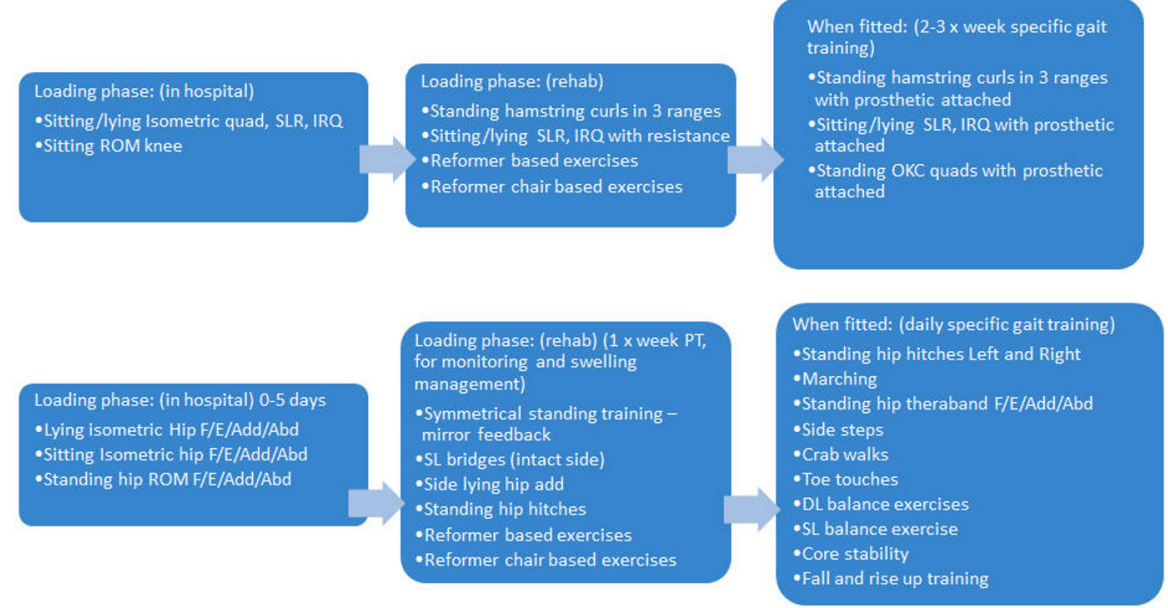

Hip Exercises

Figure 11 Transtibial osseointegration physiotherapy protocol. 




Figure 12 After fitting of prosthetic limb in a short residuum tibia.

sleeve and bushing to the dual cone using an external screw, all the time securing the implant to prevent rotation using a special device (figures 8 and 9 ).

\section{Postoperative rehabilitation}

The rehabilitation for transtibial osseointegration is carried out in phases and described in details in figures 10 and 11 . The adherence to the rehabilitation protocol is recorded in the database by the physiotherapist. Following the fitting of a prosthetic limb (figure 12), patients are encouraged to weight-bear daily on their prosthesis using two crutches for 6 weeks and then one crutch on the opposite side for a further 6 weeks and then unaided thereafter.

\section{Outcome}

\section{Data sampling}

Data sampling is done at baseline preoperatively and postoperatively at 3,6 and 12 months and yearly follow-ups thereafter. It is done by dedicated research assistants who are unaware of the details of patients' demographic characteristics, surgical and implant details and previous scores to reduce the risk of any bias. Clinical information from surgery and follow-ups are added to the database by the operating or reviewing surgeon. Data that are sampled including the time points of measurement are tabulated in table 2.

\section{Adverse events}

Adverse events are reported which includes infection that require administration of intravenous or oral antibiotics or surgical intervention, periprosthetic fracture, implant breakage, aseptic loosening, need for revision surgery or additional amputation and death. Severity of infections are assessed and graded according to $\mathrm{Al}$ Muderis et al classification system. ${ }^{25}$

\section{Data analysis}

The primary questions this study aim to identify are (1) the individual patient characteristics or factors that have a positive or negative influence in the outcomes measured or in other words who would be most or least benefitted with this novel procedure and who would be at a higher or lower risk of failure? (2) What are the rates of additional intervention for patients undergoing Transtibial
Osseointegration (TTOI), and for what reasons? This project will also aim to collect data which can allow investigation of diverse questions regarding transtibial osseointegration as further insight develops.

The influence of various factors such as patient gender, age and cause of amputation on dependent variables relating to potential risks (infection, fracture, further surgery, etc) or benefit (mobility, Quality of Life (QOL) outcomes, etc) will be assessed. Multivariable logistic regression will be performed with a focus to identify factors associated with further surgery, specifically for infection, periprosthetic fracture, implant fracture and aseptic loosening. Additionally, factors associated with Daily prosthesis wear hours, Prosthetic wear satisfaction, SF-36 and mobility (6MWT, TUG, K-level) will be evaluated. Separate regression models will be developed for short and long residuum TTOIs as well. A $p$ value of $\leq 0.05$ will be considered as significant. The $p$ value for each regression identifying significant predictors of dependent variable outcome will be reported, as will the coefficients of relative influence of each variable.

The preoperative versus postoperative continuous value data will be presented as mean and SD and compared with Student's t-test or analysis of variance if the data are normally distributed. Post-hoc analyses related to longitudinal data analysis at T0, T1, T2 and so on will also be performed. Should the data not be normally distributed the median and Inter-Quartile Ranges will be reported and comparison made using Wilcoxon test.

\section{Reducing risk of bias}

In addition to reducing the risk of selection bias as described earlier, bias relating to surgeon expertise and protocol adherence is eliminated since all operations will be performed by a single primary surgeon. Bias related to data collection will be minimised by employing dedicated research assistants who will be unaware about details of patient demographic characteristics, surgical and implant details and previous recorded scores. Further, the results of functional outcome measures (6MWT, TUG, K-levels) depend on the patients' actual performance, while the results of subjective outcome measures are completely patient reported from surveys. In addition, the assessors will not be involved in data analysis.

\section{Patient and public involvement}

Patients or the public were not involved in the design, or conduct, or reporting, or dissemination plans of our research.

\section{Ethics and dissemination}

The ethics approval for the study has been received from the University of Notre Dame, Sydney, Australia (0 14153S). All patients included in this study will sign a consent form that provides sufficient information about the study for patients to make an informed decision about their participation. Outcomes of the current study 
Table 2 Data sampling table showing the parameters sampled and time points of measurement

Parameter sampled

Details

Name

Date of birth

Address

Phone number/email

Gender

Height

Weight

Military

Athlete

Race

Education level

Employment status before OI surgery

Type of occupation before OI surgery

Age at first surgery

Date of first surgery

Any further surgeries

Side

Bilateral

Mixed

Same day amputation and $\mathrm{OI}$

Cause of amputation

Date of amputation

Comorbidities

Psychiatric evaluation before surgery

Depression

Alcohol >3/day

TMR at index surgery

Reasons for osseointegration

Implant details

Retention of hardware

Implant removal

Reason for removal

Years to fail

Re-implant date

Further surgeries details

Antibiotics administration

Other adverse events

Length of residuum

Length after $\mathrm{OI}$

Pre-op weight bearing status

Pre-op K-Level

Yes/No

Yes/No

Yes/No

Yes/No number length
Time point of
measurement

TO

TO

TO

T0

TO

TO

TO

TO

TO

TO

TO

TO

TO

TO

TO

Yes/No. Dates of further surgeries if yes

When it occurs

TO

TO

TO

TO/TS

Each cause assigned a number $\quad$ T0

TO

Each cause assigned a number $\quad$ T0

Yes/No TO

Yes/No T0

Yes/No TO

Yes/No T0

Fit problems/skin problems/painful prosthesis/prosthetic mobility T0 dissatisfaction/other pain/other causes. Each cause assigned a

Implant brand, type, manufacture method, collared/flared, width, TS

None/cable/screw/both

When it occurs

When it occurs

When it occurs

When it occurs

Washouts/neurectomy/refashioning/periprosthetic fractures/other When it occurs surgeries details

Intravenous/oral. Details

When it occurs

When it occurs

TO

TS

T0

TO 
Table 2 Continued

\section{Parameter sampled}

Pre-op walking aid

Pre-op 6-Minute Walk Test

Pre-op Timed Up-and-Go Test

Pre-op SF-36 (PCS)

Pre-op SF-36 (MCS)

Pre-op subjective
Details

Time point of measurement

Pre-op stump pain (VAS) level of function with your current prosthesis?'

\begin{tabular}{|c|c|c|}
\hline \multicolumn{2}{|l|}{ Daily prosthetic wear hours } & TO \\
\hline \multicolumn{2}{|l|}{ Prosthetic wear satisfaction } & T0 \\
\hline Adherence to rehabilitation protocol & Yes/No & TR \\
\hline \multicolumn{2}{|l|}{ Post-op weight bearing status } & $\mathrm{T} 1, \mathrm{~T} 2, \mathrm{~T} 3, \mathrm{~T} 4 \ldots$ \\
\hline \multicolumn{2}{|l|}{ Post-op K-level } & $\mathrm{T} 1, \mathrm{~T} 2, \mathrm{~T} 3, \mathrm{~T} 4 \ldots$ \\
\hline \multicolumn{2}{|l|}{ Post-op walking aid } & $\mathrm{T} 1, \mathrm{~T} 2, \mathrm{~T} 3, \mathrm{~T} 4 \ldots$ \\
\hline \multicolumn{2}{|l|}{ Post-op 6-Minute Walk Test } & $\mathrm{T} 1, \mathrm{~T} 2, \mathrm{~T} 3, \mathrm{~T} 4 \ldots$ \\
\hline \multicolumn{2}{|l|}{ Post-op Timed Up-and-Go Test } & $\mathrm{T} 1, \mathrm{~T} 2, \mathrm{~T} 3, \mathrm{~T} 4 \ldots$ \\
\hline \multicolumn{2}{|l|}{ Post-op SF-36 (PCS) } & $\mathrm{T} 1, \mathrm{~T} 2, \mathrm{~T} 3, \mathrm{~T} 4 \ldots$ \\
\hline \multicolumn{2}{|l|}{ Post-op SF-36 (MCS) } & $\mathrm{T} 1, \mathrm{~T} 2, \mathrm{~T} 3, \mathrm{~T} 4 \ldots$ \\
\hline Post-op subjective & $\begin{array}{l}\text { Functional level and problems. 'How would you summarise your } \\
\text { level of function with your current prosthesis?' }\end{array}$ & $\mathrm{T} 1, \mathrm{~T} 2, \mathrm{~T} 3, \mathrm{~T} 4 \ldots$ \\
\hline \multicolumn{2}{|l|}{ Post-op stump Pain (VAS) } & $\mathrm{T} 1, \mathrm{~T} 2, \mathrm{~T} 3, \mathrm{~T} 4 \ldots$ \\
\hline \multicolumn{2}{|l|}{ Daily prosthetic wear hours } & $\mathrm{T} 1, \mathrm{~T} 2, \mathrm{~T} 3, \mathrm{~T} 4$ \\
\hline \multicolumn{2}{|l|}{ Prosthetic wear satisfaction } & $\mathrm{T} 1, \mathrm{~T} 2, \mathrm{~T} 3, \mathrm{~T} 4$ \\
\hline
\end{tabular}

T0: preoperative, TS: at surgery, TR: during rehabilitation, T1: 3 months, T2: 6 months, T3: 1 year, T4: 2 years and so on. OI: Osseointegration, TMR: Targeted Muscle Reinnervation, PCS: Physical Component Score, MCS: Mental Component Score, VAS: Visual Analog Scale

will be disseminated by publications in peer-reviewed academic journals and presentations at relevant orthopaedic conferences.

\section{DISCUSSION}

This study will be the first major one to focus on transtibial osseointegration only and will have the largest cohort reported in literature so far. The findings of the study would assess whether osseointegration in TTA is feasible in terms of risks and benefits and also make an important contribution to the otherwise limited literature regarding outcomes of osseointegration in lower extremity amputations. As evidenced by literature, TTA using TSP suffer from same difficulties involving skin breakdown, ${ }^{8}$ suboptimal fit ${ }^{49}$ and pain ${ }^{4}$ as do the transfemoral ones, which ultimately affect their prosthetic use, mobility and overall quality of life. As the dramatically different concept of osseointegration proved life-changing in management of TFA with established safety, it is only logical to extend the science to TTA and document the outcomes.
The challenges posed by TSP were overcome by direct anchorage of the implant to the bone that enabled physiological weight bearing, ${ }^{17}$ increased flexibility and range of motion, ${ }^{50}$ sitting comfort, ${ }^{51}$ mechanoreception-based sensory feedback (osseoperception), ${ }^{23}$ improved donning and doffing, ${ }^{24}$ better mobility ${ }^{7}$ and improved prosthetic use, ${ }^{24}$ body image ${ }^{49}$ and quality of life. ${ }^{24}$ The safety of the implant was established in subsequent studies in terms of stability and risk of infection. ${ }^{25}$

Although largely unreported in literature so far, the further application of osseointegration to TTA has been done in pilot project by our group to suitable patients as well as some other surgeons worldwide. Prospective outcomes at 12 months of five patients with peripheral vascular disease who underwent transtibial osseointegration was published recently by $\mathrm{Al}$ Muderis et al. ${ }^{43}$ Results showed that all the patients enrolled in the study were able to mobilise unaided at final follow-up. There was notable improvement of objective functional measures of 6MWT and TUG as well as subjective functional measures, while only two superficial infections were noted which resolved 
with conservative treatment and no implant loosening or other adverse event documented. However, two previous studies from Germany ${ }^{26}{ }^{28}$ reporting on nine individuals with transtibial amputations treated with their custom cobalt chrome implants reported an explanation rate of $43 \%$ and rates of both septic and aseptic loosening of $22 \%$ each, though patient eligibility, rehabilitation and follow-up is unclear.

Recently, another study comprising a small number of nine transtibial patients having a follow-up of only 12 months has been reported from The Netherlands. ${ }^{44}$ The cohort was a mixed one with majority (31 patients) being transfemoral patients. Comparison of outcomes between transtibial and transfemoral osseointegrated patients revealed higher overall baseline values in transtibial patients except walking distance in daily life and prosthetic comfort. Improvement in the outcome measures was also greater in transtibial patients (except hip abductor strength and prosthesis wearing time), and at final follow-up lesser transtibial patients experienced stump pain as compared with transfemoral patients (transfemoral: 20/31 (65\%), transtibial: 2/9 (22\%)). Major adverse events related to implants was recorded as $8 \%$ which included both groups and included three dualcone breakages and four bone fractures (due to fall), which were all managed successfully. However, a lower uneventful course was noted in transtibial patients $(44 \%)$ compared with transfemoral ones $(61 \%)$. The authors concluded that transtibial osseointegration was both efficacious and safe at 12 months follow-up.

Thus, the proposed study would comprise the largest cohort of TTA undergoing osseointegration with a substantial follow-up time. The clinical outcomes, adverse events, and their associations noted in this study would help considerably to set the standard of care in transtibial amputee patients and provide directions of further research in terms of implant design, surgical technique, rehabilitation or management of complications.

\section{Author affiliations}

${ }^{1}$ Department of Orthopaedic Surgery, Macquarie University Hospital, North Ryde BC, New South Wales, Australia

${ }^{2}$ The Limb Reconstruction Discipline, Macquarie University Hospital, North Ryde BC, New South Wales, Australia

${ }^{3}$ Faculty of Medicine and Health, The University of Sydney, Sydney, New South Wales, Australia

${ }^{4}$ Department of Orthopaedic Surgery, NHS Fife, Kirkcaldy, UK

${ }^{5}$ Department of Clinical Medicine, Faculty of Medicine, Health and Human Sciences, Macquarie University, Sydney, New South Wales, Australia

Acknowledgements The authors thank Bridget Dean for formulating transtibial rehabilitation and physiotherapy protocol.

Contributors RH: study design; patient care and surgical team; manuscript preparation. SA-J: data collection; patient care and surgical team. JH: data collection; patient care and surgical team. MAA: data collection; patient care and surgical team. KD: data collection; patient care and surgical team. YCT: data collection, statistical evaluation. WY-RL: data collection; manuscript preparation. CR: patient care; data collection; manuscript preparation. MAM: study design; patient care and surgical procedure; manuscript preparation.

Funding The authors have not declared a specific grant for this research from any funding agency in the public, commercial or not-for-profit sectors.
Competing interests MAM receives royalties for design contributions for the Osseointegrated Prosthetic Limb (OPL; Permedica s.p.a; Milan, Italy) implant system.

Patient and public involvement Patients and/or the public were not involved in the design, or conduct, or reporting, or dissemination plans of this research.

Patient consent for publication Not required.

Provenance and peer review Not commissioned; externally peer reviewed.

Open access This is an open access article distributed in accordance with the Creative Commons Attribution Non Commercial (CC BY-NC 4.0) license, which permits others to distribute, remix, adapt, build upon this work non-commercially, and license their derivative works on different terms, provided the original work is properly cited, appropriate credit is given, any changes made indicated, and the use is non-commercial. See: http://creativecommons.org/licenses/by-nc/4.0/.

\section{ORCID iD}

William Yenn-Ru Lu http://orcid.org/0000-0001-6919-8367

\section{REFERENCES}

1 Hagberg K, Brånemark R. Consequences of non-vascular transfemoral amputation: a survey of quality of life, prosthetic use and problems. Prosthet Orthot Int 2001;25:186-94.

2 Hoyt BW, Walsh SA, Forsberg JA. Osseointegrated prostheses for the rehabilitation of amputees (OPRA): results and clinical perspective. Expert Rev Med Devices 2020;17:17-25.

3 Thurston AJ. Paré and prosthetics: the early history of artificial limbs. ANZ J Surg 2007;77:1114-9.

4 Zaid MB, O'Donnell RJ, Potter BK, et al. Orthopaedic osseointegration: state of the art. J Am Acad Orthop Surg 2019;27:e977-85.

5 Demet K, Martinet N, Guillemin F, et al. Health related quality of life and related factors in 539 persons with amputation of upper and lower limb. Disabil Rehabil 2003;25:480-6.

6 Marx RG, Wilson SM, Swiontkowski MF. Updating the assignment of levels of evidence. J Bone Joint Surg Am 2015;97:1-2.

7 Van de Meent H, Hopman MT, Frölke JP. Walking ability and quality of life in subjects with Transfemoral amputation: a comparison of Osseointegration with socket prostheses. Arch Phys Med Rehabil 2013:94:2174-8.

8 Dudek NL, Marks MB, Marshall SC, et al. Dermatologic conditions associated with use of a lower-extremity prosthesis. Arch Phys Med Rehabil 2005;86:659-63.

9 Ghoseiri K, Safari MR. Prevalence of heat and perspiration discomfort inside prostheses: literature review. J Rehabil Res Dev 2014;51:855-68.

10 Lyon CC, Kulkarni J, Zimerson E, et al. Skin disorders in amputees. J Am Acad Dermatol 2000;42:501-7.

11 Meulenbelt HE, Geertzen JH, Jonkman MF, et al. Determinants of skin problems of the stump in lower-limb amputees. Arch Phys Med Rehabil 2009;90:74-81.

12 Al Muderis MM, Lu WY, Li JJ, et al. Clinically relevant outcome measures following limb osseointegration; systematic review of the literature. J Orthop Trauma 2018;32:e64-75.

13 Hunter SW, Batchelor F, Hill KD, et al. Risk factors for falls in people with a lower limb amputation: a systematic review. $P m R$ 2017;9:170-80.

14 Leijendekkers RA, van Hinte G, Frölke JP, et al. Comparison of bone-anchored prostheses and socket prostheses for patients with a lower extremity amputation: a systematic review. Disabil Rehabil 2017;39:1045-58.

15 Amtmann D, Morgan SJ, Kim J, et al. Health-related profiles of people with lower limb loss. Arch Phys Med Rehabil 2015;96:1474-83.

16 Li Y, Brånemark R. Osseointegrated prostheses for rehabilitation following amputation: The pioneering Swedish model. Unfallchirurg 2017;120:285-92.

17 Overmann AL, Aparicio C, Richards JT, et al. Orthopaedic osseointegration: Implantology and future directions. J Orthop Res 2020;38:1445-54.

18 Adell R, Eriksson B, Lekholm U, et al. Long-term follow-up study of osseointegrated implants in the treatment of totally edentulous jaws. Int J Oral Maxillofac Implants 1990;5:347-59.

19 Brånemark PI, Adell R, Albrektsson T, et al. Osseointegrated titanium fixtures in the treatment of edentulousness. Biomaterials 1983;4:25-8. 
20 Brånemark PI, Hansson BO, Adell R, et al. Osseointegrated implants in the treatment of the edentulous jaw. Experience from a 10-year period. Scand J Plast Reconstr Surg Suppl 1977;16:1-132.

21 Brånemark R, Brånemark PI, Rydevik B, et al. Osseointegration in skeletal reconstruction and rehabilitation: a review. J Rehabil Res Dev 2001;38:175-81.

22 Hagberg K, Hansson E, Brånemark R. Outcome of percutaneous osseointegrated prostheses for patients with unilateral Transfemoral amputation at two-year follow-up. Arch Phys Med Rehabil 2014;95:2120-7.

23 Hagberg K, Häggström E, Jönsson S. Osseoperception and osseointegrated prosthetic limbs. In: MacLachlan M, Desmond D, Gallagher P, eds. Psychoprosthetics. London: Springer, 2008: 131-40.

24 Hagberg K, Brånemark R, Gunterberg B, et al. Osseointegrated trans-femoral amputation prostheses: prospective results of general and condition-specific quality of life in 18 patients at 2-year followup. Prosthet Orthot Int 2008;32:29-41.

25 Al Muderis M, Khemka A, Lord SJ, et al. Safety of osseointegrated implants for Transfemoral amputees: a two-center prospective cohort study. J Bone Joint Surg Am 2016;98:900-9.

26 Aschoff H-H, Juhnke D-L. [Endo-exo prostheses : Osseointegrated percutaneously channeled implants for rehabilitation after limb amputation]. Unfallchirurg 2016;119:421-7.

27 Brånemark R, Berlin O, Hagberg K, et al. A novel osseointegrated percutaneous prosthetic system for the treatment of patients with Transfemoral amputation: a prospective study of 51 patients. Bone Joint J 2014;96-B:106-13.

28 Juhnke D-L, Aschoff H-H. [Endo-exo prostheses following limbamputation]. Orthopade 2015;44:419-25.

29 Juhnke D-L, Beck JP, Jeyapalina S, et al. Fifteen years of experience with Integral-Leg-Prosthesis: cohort study of artificial limb attachment system. J Rehabil Res Dev 2015;52:407-20.

30 Muderis MA, Tetsworth K, Khemka A, et al. The osseointegration group of Australia accelerated protocol (OGAAP-1) for two-stage osseointegrated reconstruction of amputated limbs. Bone Joint $J$ 2016;98-B:952-60.

31 Tillander J, Hagberg K, Berlin Örjan, et al. Osteomyelitis risk in patients with Transfemoral amputations treated with osseointegration prostheses. Clin Orthop Relat Res 2017;475:3100-8.

32 Dougherty PJ. Transtibial amputees from the Vietnam war. Twentyeight-year follow-up. J Bone Joint Surg Am 2001;83:383-9.

33 Harness N, Pinzur MS. Health related quality of life in patients with dysvascular transtibial amputation. Clin Orthop Relat Res 2001;383:204-7.

34 Knežević A, Salamon T, Milankov M, et al. Assessment of quality of life in patients after lower limb amputation. Med Pregl 2015;68:103-8.

35 Dillingham TR, Pezzin LE, MacKenzie EJ. Limb amputation and limb deficiency: epidemiology and recent trends in the United States. South Med J 2002;95:875-83.

36 Ziegler-Graham K, MacKenzie EJ, Ephraim PL, et al. Estimating the prevalence of limb loss in the United States: 2005 to 2050. Arch Phys Med Rehabil 2008;89:422-9.
37 Walker CR, Ingram RR, Hullin MG, et al. Lower limb amputation following injury: a survey of long-term functional outcome. Injury 1994;25:387-92.

38 Hoaglund FT, Jergesen HE, Wilson L, et al. Evaluation of problems and needs of veteran lower-limb amputees in the San Francisco Bay area during the period 1977-1980. J Rehabil R D 1983;20:57-71.

39 Behr J, Friedly J, Molton I, et al. Pain and pain-related interference in adults with lower-limb amputation: comparison of kneedisarticulation, transtibial, and Transfemoral surgical sites. J Rehabil Res Dev 2009;46:963-72.

40 Dillingham TR, Pezzin LE, MacKenzie EJ, et al. Use and satisfaction with prosthetic devices among persons with trauma-related amputations: a long-term outcome study. Am J Phys Med Rehabil 2001;80:563-71.

41 Pezzin LE, Dillingham TR, Mackenzie EJ, et al. Use and satisfaction with prosthetic limb devices and related services. Arch Phys Med Rehabil 2004:85:723-9.

42 Butler K, Bowen C, Hughes A-M, et al. A systematic review of the key factors affecting tissue viability and rehabilitation outcomes of the residual limb in lower extremity traumatic amputees. J Tissue Viability 2014;23:81-93.

43 Atallah R, Li JJ, Lu W, et al. Osseointegrated transtibial implants in patients with peripheral vascular disease: a multicenter case series of 5 patients with 1-year follow-up. J Bone Joint Surg Am 2017;99:1516-23.

44 Leijendekkers RA, van Hinte G, Frölke JP, et al. Functional performance and safety of bone-anchored prostheses in persons with a Transfemoral or transtibial amputation: a prospective one-year follow-up cohort study. Clin Rehabil 2019;33:450-64.

45 Lin S-J, Bose NH. Six-minute walk test in persons with transtibial amputation. Arch Phys Med Rehabil 2008;89:2354-9.

46 Schoppen T, Boonstra A, Groothoff JW, et al. The Timed "up and go" test: reliability and validity in persons with unilateral lower limb amputation. Arch Phys Med Rehabil 1999;80:825-8.

47 Al Muderis M, Lu W, Tetsworth K, et al. Single-stage osseointegrated reconstruction and rehabilitation of lower limb amputees: the osseointegration group of Australia accelerated Protocol-2 (OGAAP-2) for a prospective cohort study. BMJ Open 2017;7:e013508

48 Ware JE, Sherbourne CD. The MOS 36-item short-form health survey (SF-36). I. conceptual framework and item selection. Med Care 1992;30:473-83.

49 Muderis MA, Lu W, Glatt V, et al. Two-stage osseointegrated reconstruction of post-traumatic unilateral Transfemoral amputees. Mil Med 2018;183:496-502.

50 Tranberg R, Zügner R, Kärrholm J. Improvements in hip- and pelvic motion for patients with osseointegrated trans-femoral prostheses. Gait Posture 2011;33:165-8.

51 Hagberg K, Häggström E, Uden M, et al. Socket versus boneanchored trans-femoral prostheses: hip range of motion and sitting comfort. Prosthet Orthot Int 2005;29:153-63. 